ISSN 0103-9954

\title{
COMPOSIÇÃO E EFICIÊNCIA DA UTILIZAÇÃO BIOLÓGICA DE NUTRIENTES EM FRAGMENTO DE MATA ATLÂNTICA EM PERNAMBUCO
}

\section{COMPOSITION AND NUTRIENT BIOLOGICAL UTILIZATION EFFICIENCY IN ATLANTIC FOREST FRAGMENT IN PERNAMBUCO, BRAZIL}

\author{
Silvana Andreoli Espig ${ }^{1}$ Fernando José Freire ${ }^{2}$ Luiz Carlos Marangon ${ }^{3}$ \\ Rinaldo Luiz Caraciolo Ferreira ${ }^{4}$ Maria Betânia Galvão dos Santos Freire ${ }^{5}$ Darci Bacelar Espig ${ }^{6}$ \\ RESUMO
}

A maioria dos estudos sobre teores de nutrientes em matas naturais se concentra na serapilheira. No Brasil, em Mata Atlântica, foram realizadas pesquisas na Mata de Dois Irmãos/PE, na Floresta da Tijuca/RJ e na Mata Salão Dourado/MG. Neste trabalho, a área em estudo constitui um remanescente de Mata Atlântica, enquadrando-se como Floresta Estacional Semidecidual. O trabalho objetivou determinar, por meio da fitossociologia, as dez espécies de maior valor de importância (VI), avaliar os teores dos nutrientes Ca, Mg, $\mathrm{P}, \mathrm{K}$ e $\mathrm{N}$ nas folhas dessas espécies, estimar sua biomassa foliar e determinar a eficiência de utilização biológica desses nutrientes, ampliando assim, o conhecimento sobre os remanescentes de Mata Atlântica no Brasil. Metodologicamente, depois de definidas as dez espécies de maior VI, amostraram-se os indivíduos de maior circunferência a 1,30 m do solo (CAP) de cada espécie, e coletaram-se as folhas da parte intermediária da copa, formando uma amostra composta em que foram determinados os teores de Ca, Mg, P, K e N. Neste estudo, estimou-se a biomassa foliar em cada espécie e para transformar essa biomassa em $\mathrm{kg} / \mathrm{ha}$, somaramse as biomassas foliares dos indivíduos da espécie encontrados na área. O conteúdo de nutrientes na biomassa foliar das espécies ( $\mathrm{kg} / \mathrm{ha}$ ) foi obtido multiplicando-se o teor $(\mathrm{g} / \mathrm{kg})$ pela biomassa foliar $(\mathrm{kg} / \mathrm{ha})$ de cada espécie. A eficiência de utilização biológica foi calculada pela razão entre a biomassa foliar e o conteúdo de nutrientes das espécies. Os teores dos nutrientes, nas folhas, apresentaram a seguinte ordem decrescente $\mathrm{N}>\mathrm{Ca}>\mathrm{K}>\mathrm{Mg}>\mathrm{P}$, porém em algumas espécies o teor de $\mathrm{K}$ foi maior do que o de Ca. A biomassa foliar das dez espécies de maior VI foi de 3,3 $\mathrm{t} \mathrm{ha}^{-1}$, e representaram 24,5\% da biomassa foliar total (13,4 t $\mathrm{ha}^{-1}$ ) do fragmento. A espécie Schefflera morototoni (Aubl.) Maguire, Steyerm \& Frodin foi responsável pelo maior aporte via foliar de Ca e Mg e Helicostylis tomentosa (Poepp. \& Endl.) J.F., Macbr. pelo de P, K e N no fragmento. A eficiência de utilização de nutrientes apresentou a seguinte ordem $\mathrm{P}>\mathrm{Mg}>\mathrm{K}>\mathrm{Ca}>\mathrm{N}$.

Palavras-chave: floresta nativa; biomassa foliar; teor de nutrientes.

\section{ABSTRACT}

The vast majority of research on nutrients in natural forest is concentrated in the litter. In Brazil, in Atlantic Forest, some investigations have been conducted in few locations such as Dois Irmãos Forest (PE), Tijuca Forest (RJ) and Salão Dourado Forest (MG). In the present research, the studied area was characterized as remaining of Atlantic Forest classified as slope, ombrofilous forest. The objective was to determine by a phytosociologic study the ten species of most important value (VI) and to evaluate the Ca, $\mathrm{Mg}, \mathrm{P}, \mathrm{K}$ and $\mathrm{N}$ nutrients contents in these species leaves, likewise to estimate leaf biomass and the

1. Engenheira Florestal, MSc., Doutoranda do Instituto Nacional de Pesquisas Espaciais (INPE). Av. dos Astronautas, 1.758, Jd. Granja, CEP: 12227-010, Sâo José dos Campos (SP). sil.andreoli@gmail.com

2. Engenheiro Agrônomo, DSc., Professor Adjunto da Universidade Federal Rural de Pernambuco (UFRPE). Rua Dom Manoel de Medeiros, Campus Universitário de Dois Irmãos, CEP 52191-900, Recife (PE). f.freire@depa.ufrpe.br

3. Engenheiro Florestal, DSc., Professor Associado da Universidade Federal Rural de Pernambuco (UFRPE). Rua Dom Manoel de Medeiros, Campus Universitário de Dois Irmãos, CEP 52191-900, Recife (PE). marangon@dcfl.ufrpe.br

4. Engenheiro Florestal, DSc., Professor Associado da Universidade Federal Rural de Pernambuco (UFRPE). Rua Dom Manoel de Medeiros, Campus Universitário de Dois Irmãos, CEP 52191-900, Recife (PE). rinaldo@dcfl.ufrpe.br

5. Engenheira Agrônoma, DSc., Professora Adjunto da Universidade Federal Rural de Pernambuco (UFRPE). Rua Dom Manoel de Medeiros, Campus Universitário de Dois Irmãos, CEP 52191-900, Recife (PE). betania@depa.ufrpe.br

6. Engenheiro Agrônomo, Estagiário da Universidade Federal Rural de Pernambuco (UFRPE). Rua Dom Manoel deMedeiros, Campus Universitário de Dois Irmãos, CEP 52191-900, Recife (PE). despig@uol.com.br

Recebido para publicação em 23/01/2008 e aceito em 11/08/2008. 
efficiency of biological nutrient utilization, increasing the knowledge about Atlantic Forest residues in Brazil. After determining the ten species of most important value, the largest circumference individuals at $1.30 \mathrm{~m}$ from soil surface (CAP) in each specie were sampled and the leaves from the intermediate part of the trees top were collected, making a composed sample, being determined $\mathrm{Ca}, \mathrm{Mg}, \mathrm{P}, \mathrm{K}$ and $\mathrm{N}$ contents. In this study, the leaf biomass was estimate in each species and, to transform this biomass in $\mathrm{kg} / \mathrm{ha}$, the leaf biomasses from the species' individuals were added in the area. The nutrient contents in leaf biomass from the species $(\mathrm{kg} / \mathrm{ha})$ were achieved multiplying the content $(\mathrm{g} / \mathrm{kg})$ by leaf biomass $(\mathrm{kg} / \mathrm{ha})$ in each specie. The biological utilization efficiency was calculated considering the ratio between the leaf biomass and the nutrient contents from species. The leaves nutrient contents were in a decreasing order $\mathrm{N}>\mathrm{Ca}>\mathrm{K}>\mathrm{Mg}>\mathrm{P}$, but in some species the $\mathrm{K}$ content was higher than $\mathrm{Ca}$ content. The leaf biomass of the ten most important value species was $3.3 \mathrm{t} \mathrm{ha}^{-1}$ which represented $24.5 \%$ of the total spot leaf biomass $\left(13.4 \mathrm{t} \mathrm{ha}^{-1}\right)$. Schefflera morototoni (Aubl.) Maguire, Steyerm \& Frodin showed the most leaf entrance of Ca e Mg while Helicostylis tomentosa (Poepp. \& Endl.) J.F., Macbr. showed the best results for P, K and N in the spot. The biological utilization efficiency presented the sequence $\mathrm{P}>\mathrm{Mg}>\mathrm{K}>\mathrm{Ca}>\mathrm{N}$.

Keywords: native forest; leaf biomass; leaf nutrient content.

\section{INTRODUÇÃO}

A maioria dos estudos sobre teores de nutrientes em matas naturais se concentra na serapilheira e em povoamentos florestais, sobretudo de eucalipto, nos compartimentos vegetais e na serapilheira (REIS e BARROS, 1990). Nas florestas tropicais naturais e plantadas, pesquisas recentes descorem sobre os teores de nutrientes nos diversos componentes da vegetação (SCHUMACHER e CALDEIRA, 2001; BALIEIRO et al., 2004; VITAL et al., 2004; SILVEIRA et al., 2007; SCHUMACHER et al., 2008). Entretanto, trabalhos de referência em matas naturais tropicais úmidas foram realizados por Golley et al. (1978), no Panamá.

No Brasil, na Mata Atlântica, foram realizadas pesquisas na Mata de Dois Irmãos/PE (SAMPAIO et al., 1988); na Floresta da Tijuca/RJ (CLEVELÁRIO JÚNIOR, 1996), na Mata Salão Dourado, na região do Médio Rio Doce/MG (DRUMOND et al., 1997), na Mata do Bexinguento/RJ (VITAL et al., 2004). Nesses fragmentos, os teores de nutrientes encontrados nas folhas foram $\mathrm{N}>\mathrm{K}>\mathrm{Ca}>\mathrm{Mg}>\mathrm{P}$. Porém, Drumond et al. (1997) encontraram teores de Ca maiores do que os de K e Oliveira (1997) encontrou retorno de macronutrientes na serapilheira na seguinte ordem decrescente $\mathrm{Ca}>\mathrm{N}>\mathrm{K}>\mathrm{Mg}>\mathrm{P}$.

Na matéria seca dos vegetais, podem-se encontrar diferenças nos teores dos nutrientes que podem ser reflexo da influência da fertilidade do solo (VITOUSEK e SANFORD, 1986). Assim, os teores dos nutrientes foliares têm sido utilizados como indicadores do estado nutricional da planta (VITOUSEK e SANFORD, 1986), podendo-se, com eles e com a biomassa, calcular-se a eficiência nutricional das espécies de um povoamento florestal.

A eficiência nutricional das espécies é a capacidade de absorção e/ou de utilização de nutrientes, sendo específica do nutriente considerado (GRAHAM, 1984). Assim, a eficiência nutricional das espécies comerciais plantadas é mais pesquisada como Eucalyptus sp. (BARROS et al., 1985; MOLICA, 1992) e Mimosa caesalpiniifolia (Benth.) (MOURA et al., 2006a,b), pela importância dessa informação no manejo das adubações. No entanto, Drumond et al.(1997) avaliaram a eficiência de utilização de nutrientes nas dez espécies florestais da Mata Atlântica de maior valor de importância e encontraram que essa eficiência foi $\mathrm{P}>\mathrm{Mg}>\mathrm{K}>\mathrm{Ca}>\mathrm{N}$.

Nesse contexto, pesquisando um fragmento de Mata Atlântica em Pernambuco, este trabalho objetivou determinar, por meio da fitossociologia, as dez espécies de maior valor de importância, avaliar os teores dos nutrientes $\mathrm{Ca}, \mathrm{Mg}, \mathrm{K}, \mathrm{P}$ e $\mathrm{N}$ nas folhas dessas espécies, estimar sua biomassa foliar e determinar a eficiência de utilização biológica desses nutrientes, ampliando assim o conhecimento sobre os remanescentes de Mata Atlântica no Brasil.

\section{MATERIAL E MÉTODO}

A área em estudo constitui um remanescente de Mata Atlântica, enquadrando-se como Floresta Ombrófila Densa (VELOSO e GÓES FILHO, 1982) que, segundo o IBGE (1992), se classifica como Floresta Estacional Semidecidual. O solo no fragmento foi classificado como LATOSSOLO AMARELO distrófico (EMBRAPA, 1999b). 
Para o estudo fitossociológico, fez-se uma amostragem sistemática, utilizando-se o método de parcelas fixas, as quais foram plotadas em transeções paralelas distando entre si $10 \mathrm{~m}$, e entre unidades amostrais, na mesma transeção, distando $25 \mathrm{~m}$. Delimitou-se a área de 1,0 ha para a realização do estudo fitossociológico, demarcando-se quarenta parcelas de 10 x 25 m (MARANGON, 1999) e considerando-se um efeito de borda de $20 \mathrm{~m}$.

Após a demarcação das parcelas, fizeram-se a mensuração da circunferência a 1,30 m do solo (CAP) nos indivíduos com CAP $\geq 15,0 \mathrm{~cm}$ e a classificação das espécies, segundo Cronquist (1988). Com esses dados, calcularam-se as estimativas da estrutura horizontal que definiram as espécies de maior valor de importância (VI). Essas estimativas foram calculadas de acordo com as seguintes expressões:

$$
D A=\frac{n i}{A} \text { e } D R=\frac{n i}{N} \times 100
$$

Em que $D A$ é a densidade absoluta (indivíduos/ha); $D R$ é a densidade relativa (\%); ni é o número de indivíduos da espécie i; $A$ é a área (ha); e $N$ é o número total de indivíduos amostrados na área.

$$
F A=\frac{U}{U T} \times 100 \text { e } F R=\frac{F A}{\sum F A} \times 100
$$

Em que $F A$ é a freqüência absoluta (\%); $F R$ é a freqüência relativa (\%); $U$ é o número de unidades amostrais em que ocorreu a espécie i; e UT é o número total de unidades amostrais.

$$
D o A=\frac{A B}{A} \text { e } D o R=\frac{A B}{A B T} \times 100
$$

Em que $D o A$ é a dominância absoluta ( $\left.\mathrm{m}^{2} / \mathrm{ha}\right)$; $D o R$ é a dominância relativa (\%); $A B$ é a área basal da espécie i $\left(\mathrm{m}^{2}\right)$; e $A B T$ é a área basal total de todas as espécies $\left(\mathrm{m}^{2}\right)$.

Assim, o valor de importância (VI) foi calculado de acordo com a seguinte expressão:

$V I=D R+F R+D o R$ (\%).

Em que $D R$ é a densidade relativa (\%); FR é a freqüência relativa (\%); e $D o R$ é a dominância relativa expressão:

Calculou-se também o índice de diversidade de Shanonn \& Weaver $\left(\mathrm{H}^{\prime}\right)$, de acordo com a seguinte

$$
H^{\prime}=-\sum_{i=1}^{N} p i \ln p i
$$

Em que $H^{\prime}$ é o índice de diversidade de Shanonn \& Weaver (nats/indivíduos); $p i=\frac{n i}{N} ; n i$ é o número de indivíduos da espécie i; $N$ é o número total de indivíduos amostrados na área; e ln é o logaritmo neperiano.

Após definirem-se as dez espécies de maior valor de importância (VI) na área delimitada, amostraram-se os três indivíduos de maior CAP de cada espécie, coletando-se as folhas da parte intermediária da copa (YOUNG e CARPENTER, 1976), formando uma amostra composta. As folhas coletadas foram levadas à estufa de circulação forçada de ar, à temperatura de $65^{\circ} \mathrm{C}$ até peso constante (MALAVOLTA et al., 1989) e, posteriormente, moídas e armazenadas.

Os nutrientes Ca, Mg, P e K foram extraídos por digestão nítrico-perclórica e o $\mathrm{N}$ total por digestão sulfúrica (BATAGLIA et al., 1983). Posteriomente, o Ca e o Mg foram determinados por espectrofotometria de absorção atômica, o P foi dosado por colorimetria (BRAGA e DEFELIPO, 1974) e o K determinado por fotometria de chama (EMBRAPA, 1999a).

Neste estudo, estimou-se a biomassa foliar em cada espécie, conforme Golley et al. (1978). Para transformar a biomassa foliar da espécie em $\mathrm{kg} / \mathrm{ha}$, somaram-se as biomassas foliares dos indivíduos da espécie encontrados na área. O conteúdo de nutrientes na biomassa foliar das espécies $(\mathrm{kg} / \mathrm{ha})$ foi obtido multiplicando-se o teor $(\mathrm{g} / \mathrm{kg})$ pela biomassa foliar $(\mathrm{kg} / \mathrm{ha})$ de cada espécie. A eficiência de utilização biológica foi calculada pela razão entre a biomassa foliar e o conteúdo de nutrientes das espécies. 


\section{RESULTADOS E DISCUSSÃO}

Pelo levantamento fitossociológico, o fragmento estudado apresentou 970 indivíduos/ha, com 107 espécies e diversidade ( $\left.\mathrm{H}^{\prime}\right)$ de 3,66 nats/espécie. Partindo-se das estimativas da estrutura horizontal, encontraram-se os valores de importância (VI) das espécies.

As dez espécies de maior VI foram Helicostylis tomentosa (22,8\%), Parkia pendula (18,97\%), Brosimum guianense (17,85\%), Mabea occidentalis (15,67\%), Miconia albicans (14,02 \%), Dialium guianensis (12,14\%), Thyrsodium schomburgkianum (11,90\%), Tapirira guianensis (9,85\%), Lecythis pisonis (8,94\%) e Schefflera morototoni (8,75\%) (Tabela 1).

Das dez espécies de maior VI num levantamento feito por Lins \& Silva (1996), no mesmo fragmento, sete delas foram enquadradas também neste levantamento, ocupando, no entanto, posições diferentes, o que está demonstrando incremento de crescimento diamétrico (área basal) e nível de inclusão (densidade e freqüência), com o tempo.

TABELA 1: Densidades absoluta e relativa, freqüências absoluta e relativa, dominâncias absoluta e relativa das dez espécies de maiores valores de importância amostradas em um fragmento de Mata Atlântica em Pernambuco.

TABLE 1: Absolute and relative densities, absolute and relative frequencies, absolute and relative dominants of ten species most importance value of in a sample remaining Atlantic Forest in Pernambuco, Brazil.

\begin{tabular}{|c|c|c|c|c|c|c|c|}
\hline \multirow{2}{*}{ Espécie } & DA & DR & FA & FR & \multirow{2}{*}{$\begin{array}{ll}\text { DoA } \\
\mathrm{m}^{2} / \mathrm{ha}\end{array}$} & DoR & VI \\
\hline & \multicolumn{2}{|c|}{ Ind./ha } & \multicolumn{2}{|c|}{$\%$} & & \multicolumn{2}{|l|}{ DUN } \\
\hline $\begin{array}{l}\text { Helicostylis tomentosa (Poepp. \& } \\
\text { Endl) Rushy }\end{array}$ & 104 & 10,72 & 75,00 & 5,85 & $16.631,07$ & 6,23 & 22,80 \\
\hline $\begin{array}{l}\text { Parkia pendula (Willd.) Benth. Ex } \\
\text { Walp. }\end{array}$ & 62 & 6,39 & 67,50 & 5,26 & $19.507,24$ & 7,31 & 18,97 \\
\hline Brosimum guianense (Aubl.) Huber & 77 & 7,94 & 60,00 & 4,68 & $13.971,70$ & 5,24 & 17,85 \\
\hline Mabea occidentalis Benth. & 95 & 9,79 & 37,50 & 2,92 & $7.881,03$ & 2,95 & 15,67 \\
\hline Miconia albicans (Sw.) Triana & 71 & 7,32 & 72,50 & 5,65 & $2.804,03$ & 1,05 & 14,02 \\
\hline Dialium guianense Steud. & 20 & 2,06 & 35,00 & 2,73 & $19.601,01$ & 7,35 & 12,14 \\
\hline Thyrsodium schomburgkianum Benth. & 45 & 4,64 & 65,00 & 5,07 & $5.837,64$ & 2,19 & 11,90 \\
\hline Tapirira guianensis Aubl. & 21 & 2,16 & 27,50 & 2,14 & $14.773,40$ & 5,54 & 9,85 \\
\hline Lecythis pisonis Cambess. & 4 & 0,41 & 10,00 & 0,78 & $20.663,80$ & 7,75 & 8,94 \\
\hline $\begin{array}{l}\text { Schefflera morototoni (Aubl.) Maguire, } \\
\text { Steyerm. \& Frodin }\end{array}$ & 20 & 2,06 & 35,00 & 2,73 & $10.563,59$ & 3,96 & 8,75 \\
\hline
\end{tabular}

Em que: DA = Densidade Absoluta; DR = Densidade Relativa; FA = Freqüência Absoluta; FR = Freqüência Relativa; DoA = Dominância Absoluta; DoR = Dominância Relativa; VI = Valor de Importância.

A composição nutricional diferiu entre as espécies (Tabela 2). A espécie que apresentou os maiores teores de $\mathrm{N}$ foi Mabea occidentalis, porém não diferiu estatisticamente dos teores encontrados nas espécies Parkia pendula, Brosimum guianense, Lecythis pisonis e Schefflera morototoni. Com os menores teores de N identificaram-se as espécies Thyrsodium schomburgkianum e Tapirira guianensis. Para Ca e Mg, destacou-se Schefflera morototoni. Para P, nenhuma espécie apresentou teores diferentes estatisticamente. Em relação aos teores de $\mathrm{K}$, destacaram-se Helicostylis tomentosa e Brosimum guianense, que não diferiram dos teores encontrados em Tapirira guianensis e Schefflera morototoni. O menor teor foi identificado em Thyrsodium schomburgkianum.

Essa diferença nos teores e a alta diversidade de espécies no fragmento (3,66 nats/espécie) é fundamental, porque confere equilíbrio na nutrição mineral, já que espécies com maior capacidade de absorção acumulam nutrientes, para posteriormente, disponibilizá-los, por meio da ciclagem, às espécies com menor capacidade de absorção, evitando perdas por lixiviação e mantendo um sistema integrado e estável, fundamental para um ecossistema florestal sustentável.

Os teores de nutrientes nas folhas das espécies seguiram a ordem decrescente $\mathrm{N}>\mathrm{Ca}>\mathrm{K}>\mathrm{Mg}>\mathrm{P}$ (Tabela 2). Essa mesma ordem, geralmente é encontrada em florestas tropicais (GOLLEY et al., 1978; SAMPAIO et al., 1988; CLEVELÁRIO JÚNIOR, 1996; VITAL et al., 2004). No entanto, em algumas 
espécies (Helicostylis tomentosa, Parkia pendula e Lecythis pisonis), o teor de $\mathrm{K}$ foi maior do que o de Ca. Porém também Drumond et al. (1997), estudando a composição mineral e demanda nutricional de espécies florestais da Mata Atlântica, encontraram espécies em que os teores de K foram maiores do que os de Ca. Oliveira (1997) encontrou teores de Ca maiores que todos os outros nutrientes na deposição de serapilheira. Essas diferenças podem ser atribuídas ao ambiente pedológico em que essas florestais foram pesquisadas e/ou a maior capacidade de absorção de determinado nutriente em relação a outro, determinada pela menor ou maior exigência nutricional da espécie.

TABELA 2: Teores de nutrientes nas folhas ( $\mathrm{g} / \mathrm{kg}$ ) das dez espécies de maior valor de importância em um fragmento de Mata Atlântica em Pernambuco.

TABLE 2: Leaves nutrient contents $(\mathrm{g} / \mathrm{kg})$ in ten species most importance value in a remaining Atlantic Forest in Pernambuco, Brazil.

\begin{tabular}{l|c|r|r|c|c}
\hline Espécie & $\mathrm{N}$ & $\mathrm{Ca}$ & $\mathrm{Mg}$ & $\mathrm{P}$ & $\mathrm{K}$ \\
\hline Helicostylis tomentosa & $21,70 \mathrm{~B}$ & $13,80 \mathrm{~B}$ & $2,00 \mathrm{~B}$ & $1,00 \mathrm{~A}$ & $17,48 \mathrm{~A}$ \\
Parkia pendula & $22,96 \mathrm{AB}$ & $8,56 \mathrm{~B}$ & $2,30 \mathrm{~B}$ & $1,12 \mathrm{~A}$ & $10,70 \mathrm{CDE}$ \\
Brosimum guianense & $22,98 \mathrm{AB}$ & $18,93 \mathrm{~B}$ & $3,35 \mathrm{~B}$ & $0,88 \mathrm{~A}$ & $16,98 \mathrm{~A}$ \\
Mabea occidentalis & $25,52 \mathrm{~A}$ & $14,90 \mathrm{~B}$ & $3,11 \mathrm{~B}$ & $0,94 \mathrm{~A}$ & $6,93 \mathrm{DE}$ \\
Miconia albicans & $21,18 \mathrm{~B}$ & $23,38 \mathrm{AB}$ & $4,12 \mathrm{~B}$ & $0,61 \mathrm{~A}$ & $11,20 \mathrm{CD}$ \\
Dialium guianense & $20,83 \mathrm{~B}$ & $11,34 \mathrm{~B}$ & $1,59 \mathrm{~B}$ & $0,94 \mathrm{~A}$ & $10,95 \mathrm{CD}$ \\
Thyrsodium schomburgkianum & $14,25 \mathrm{C}$ & $11,39 \mathrm{~B}$ & $2,79 \mathrm{~B}$ & $0,72 \mathrm{~A}$ & $6,18 \mathrm{E}$ \\
Tapirira guianensis & $15,55 \mathrm{C}$ & $16,68 \mathrm{~B}$ & $3,31 \mathrm{~B}$ & $0,83 \mathrm{~A}$ & $13,71 \mathrm{ABC}$ \\
Lecythis pisonis & $23,71 \mathrm{AB}$ & $9,92 \mathrm{~B}$ & $2,78 \mathrm{~B}$ & $1,06 \mathrm{~A}$ & $11,96 \mathrm{BC}$ \\
Schefflera morototoni & $23,61 \mathrm{AB}$ & $37,30 \mathrm{~A}$ & $11,14 \mathrm{~A}$ & $1,37 \mathrm{~A}$ & $16,22 \mathrm{AB}$ \\
\hline Média & 21,23 & 16,62 & 3,65 & 0,95 & 12,23 \\
\hline DMS & 3,55 & 15,28 & 3,54 & 0,84 & 4,52 \\
\hline CV (\%) & 5,70 & 31,61 & 33,11 & 29,64 & 12,63 \\
\hline
\end{tabular}

Em que: Médias seguidas de mesma letra na coluna não diferem estatisticamente pelo Teste de Tukey a 5\%.

Os teores médios de $\mathrm{N}, \mathrm{Mg}$ e $\mathrm{P}$ foram de 21,23; 3,65 e 0,95 g/kg respectivamente. Esses teores foram próximos aos obtidos por Clevelário Júnior (1996) e Drumond et al. (1997). Por outro lado, o teor médio de Ca verificado neste trabalho $(16,62 \mathrm{~g} / \mathrm{kg})$ foi superior aos encontrados por esses autores (7,5 e 6,95 $\mathrm{g} / \mathrm{kg}$ respectivamente). No entanto, Golley et al. (1978) encontraram teor médio de Ca nas folhas, em floresta tropical de baixada no Panamá, de $21,2 \mathrm{~g} / \mathrm{kg}$. Do mesmo modo, o teor médio de $\mathrm{K}$ obtido neste trabalho $(12,23 \mathrm{~g} / \mathrm{kg})$ difere do encontrado por Drumond et al. (1997), que foi de 6,46 g/kg, porém foi próximo do encontrado por Golley et al. (1978) e Clevelário Júnior (1996) que encontraram 14,3 e 11,3 g/kg respectivamente. Essas diferenças e/ou semelhanças podem ser atribuídas à disponibilidade de nutrientes do solo, que interfere diretamente na absorção das espécies.

Num mesmo sítio, os teores dos nutrientes podem indicar uma eficiência de absorção diferenciada entre as espécies e, em sítios distintos, comparando-se a mesma espécie, podem indicar a qualidade do sítio. Em locais mais restritivos, as espécies tendem a apresentar uma maior capacidade de absorção, otimizando os nutrientes que são colocados a disposição.

Os teores dos nutrientes, segundo alguns autores, podem indicar a demanda nutricional das espécies, quando num mesmo sítio (VITOUSEK e SANFORD, 1986). No entanto, isso não parece ser uma questão de demanda nutricional e, sim, de uma maior eficiência de absorção das espécies, sobretudo em sítios de pouca disponibilidade de nutrientes (CLARKSON, 1985).

Estimando-se a biomassa foliar, conforme Golley et al. (1978), obteve-se a biomassa das dez espécies de maior VI e do fragmento (todas as espécies), encontrando-se 3,3 e 13,4 $\mathrm{t} \mathrm{ha}^{-1}$ respectivamente, constatando-se que a biomassa das dez espécies de maior VI, correspondeu a $24,6 \%$ da biomassa foliar do fragmento.

Assim, as biomassas das dez espécies de maior VI e do fragmento encontradas neste trabalho foram superiores às de outras florestas tropicais (GOLLEY et al., 1978; CLEVELÁRIO JÚNIOR, 1996; DRUMOND, 1996; DRUMOND et al., 1997) em razão, provavelmente, da forma de estimar a biomassa foliar. Por exemplo, Clevelário Júnior (1996) estimou baseado na relação proposta por Grubb (1977) e na 
queda de folhas reportada por Oliveira (1987). Já Drumond et al. (1997) estimaram utilizando valores percentuais médios obtidos com base no peso da matéria seca de espécies nativas plantadas (DRUMOND, 1996). Contudo, provavelmente, neste trabalho, por utilizar a estimativa de biomassa foliar proposta por Golley et al. (1978), encontraram-se valores próximos aos encontrados por esses autores em floresta tropical no Panamá, que foi de $12,0 \mathrm{t} \mathrm{ha}^{-1}$ para todas as espécies.

O conteúdo de nutrientes é resultado dos teores em função da biomassa. Observaram-se maiores conteúdos de Ca e Mg em Schefflera morototoni (Tabela 3), especialmente por esta espécie apresentar altos teores desses nutrientes (Tabela 2). Entretanto, especificamente para Ca, o conteúdo dessa espécie não diferiu de Helicostylis tomentosa, Brosimum guianense e Miconia albicans (Tabela 3); encontrou-se também maior conteúdo de $\mathrm{N}$ em Helicostylis tomentosa e Parkia pendula, que não diferiu de Brosimum guianense, porém foi diferente de todas as outras espécies de maior VI, sobretudo pelas elevadas biomassas apresentadas por essas espécies (Tabela 4). Para P, apesar do elevado conteúdo apresentado por Helicostylis tomentosa e Parkia pendula, não houve diferença estatística significativa, refletindo o comportamento apresentado pelos teores (Tabela 2). Para K, destaque para Helicostylis tomentosa, que não diferiu apenas de Brosimum guianense, resultado dos elevados teores de $\mathrm{K}$ apresentados por essa espécie (Tabela 2).

TABELA 3: Conteúdo de nutrientes $\left(\mathrm{kg} \mathrm{ha}^{-1}\right)$ na biomassa foliar das dez espécies de maior valor de importância em um fragmento de Mata Atlântica em Pernambuco.

TABLE 3: Nutrient contents $\left(\mathrm{kg} \mathrm{ha}^{-1}\right)$ in leaf biomass of ten species most importance value in remaining Atlantic Forest in Pernambuco, Brazil.

\begin{tabular}{l|c|c|c|c|c}
\hline Espécie & $\mathrm{N}$ & $\mathrm{Ca}$ & $\mathrm{Mg}$ & $\mathrm{P}$ & $\mathrm{K}$ \\
\hline Helicostylis tomentosa & $10,9 \mathrm{~A}$ & $6,9 \mathrm{AB}$ & $1,0 \mathrm{~B}$ & $0,50 \mathrm{~A}$ & $8,8 \mathrm{~A}$ \\
Parkia pendula & $10,3 \mathrm{~A}$ & $3,8 \mathrm{BC}$ & $1,0 \mathrm{~B}$ & $0,50 \mathrm{~A}$ & $4,8 \mathrm{BC}$ \\
Brosimum guianense & $9,2 \mathrm{AB}$ & $7,6 \mathrm{AB}$ & $1,3 \mathrm{~B}$ & $0,35 \mathrm{~A}$ & $6,8 \mathrm{AB}$ \\
Mabea occidentalis & 5,5CD & $3,2 \mathrm{BC}$ & $0,7 \mathrm{~B}$ & $0,20 \mathrm{~A}$ & $1,5 \mathrm{D}$ \\
Miconia albicans & $6,8 \mathrm{BC}$ & $7,5 \mathrm{AB}$ & $1,3 \mathrm{~B}$ & $0,19 \mathrm{~A}$ & $3,6 \mathrm{CD}$ \\
Dialium guianense & $6,8 \mathrm{BC}$ & $3,7 \mathrm{BC}$ & $0,5 \mathrm{~B}$ & $0,31 \mathrm{~A}$ & $3,6 \mathrm{CD}$ \\
Thyrsodium schomburgkianum & 5,2CD & $4,2 \mathrm{BC}$ & $1,0 \mathrm{~B}$ & $0,26 \mathrm{~A}$ & $2,3 \mathrm{D}$ \\
Tapirira guianensis & $4,0 \mathrm{D}$ & $4,2 \mathrm{BC}$ & $0,8 \mathrm{~B}$ & $0,21 \mathrm{~A}$ & $3,5 \mathrm{CD}$ \\
Lecythis pisonis & $4,1 \mathrm{D}$ & $1,7 \mathrm{C}$ & $0,5 \mathrm{~B}$ & $0,18 \mathrm{~A}$ & $2,0 \mathrm{D}$ \\
Schefflera morototoni & $6,5 \mathrm{CD}$ & $10,4 \mathrm{~A}$ & $3,1 \mathrm{~A}$ & $0,38 \mathrm{~A}$ & $4,5 \mathrm{C}$ \\
\hline Média & 6,9 & 5,3 & 1,1 & 0,31 & 4,1 \\
\hline DMS & 2,7 & 4,7 & 0,96 & 0,35 & 2,2 \\
\hline CV (\%) & 13,2 & 30,0 & 29,2 & 38,8 & 17,8 \\
\hline
\end{tabular}

Em que: Médias seguidas de mesma letra na coluna não diferem estatisticamente pelo Teste de Tukey a 5\%.

A informação do conteúdo de nutrientes nos compartimentos das espécies vegetais é utilizada, em florestas plantadas, para prever a exportação de nutrientes durante a remoção do (s) compartimento (s) comercial(is), visando a auxiliar o planejamento adequado das fertilizações. Em florestas naturais, como deste trabalho, essa informação pode predizer a capacidade que as espécies têm para aportar nutrientes e sua contribuição no equilíbrio do ecossistema, em especial em sítios de baixa fertilidade natural.

As espécies de maior VI apresentaram diferenças quanto à eficiência de utilização para um mesmo nutriente e entre os nutrientes (Tabela 4). Quando há eficiência de absorção e pouca conversão em biomassa foliar, a eficiência de utilização é baixa. A eficiência de absorção e utilização pode estar relacionada com a disponibilidade do nutriente no sítio ou pode ser uma característica específica da espécie. Assim, em solos menos férteis, deve-se priorizar espécies com maior eficiência de absorção e, em solos mais férteis, espécies com maior eficiência de utilização de nutrientes (MOLICA, 1992).

Esses resultados demonstraram que, neste fragmento florestal e, em geral, nos ecossistemas florestais tropicais, em que os solos são, normalmente, pouco férteis e muito intemperizados, o P é um fator limitante para o crescimento das espécies, e a eficiência de utilização desse nutriente é alta em todas as espécies, comparativamente às determinadas para os outros nutrientes (Tabela 4).

Com relação às espécies, a que apresentou menor eficiência de utilização foi a Schefflera morototoni e a de maior eficiência foi Thyrsodium schomburgkianum. 
TABELA 4: Biomassa e eficiência de utilização de nutrientes em folhas das dez espécies de maior valor de importância em um fragmento de Mata Atlântica em Pernambuco.

TABLE 4: Biomass e nutrient utilization efficiency in leaves of ten species most importance value in a remaining Atlantic Forest in Pernambuco, Brazil.

\begin{tabular}{|c|c|c|c|c|c|c|}
\hline \multirow{2}{*}{ Espécie } & Biomassa & $\mathrm{N}$ & $\mathrm{Ca}$ & Mg & $\mathrm{P}$ & $\mathrm{K}$ \\
\hline & kg/ha & \multicolumn{5}{|c|}{ kg biomassa/kg nutriente } \\
\hline Helicostylis tomentosa & $502,0 \mathrm{~A}$ & 46,1B & 72,5CD & 499,9AB & $1.000,3 \mathrm{~A}$ & $57,2 \mathrm{C}$ \\
\hline Parkia pendula & $450,1 \mathrm{AB}$ & $43,6 \mathrm{~B}$ & $116,8 \mathrm{~A}$ & 434,5ABC & $894,1 \mathrm{~A}$ & 93,4ABC \\
\hline Brosimum guianense & $400,5 B C$ & 43,5B & $52,8 \mathrm{DE}$ & 298,4 ABC & $1.131,4 \mathrm{~A}$ & $58,9 \mathrm{C}$ \\
\hline Mabea occidentalis & 213,9FG & $39,2 B$ & $67,1 \mathrm{CDE}$ & 321,9ABC & $1.062,2 \mathrm{~A}$ & $144,2 \mathrm{AB}$ \\
\hline Miconia albicans & $321,2 \mathrm{DE}$ & $47,2 B$ & 42,8EF & 242,6BC & $1.650,0 \mathrm{~A}$ & 89,3АВC \\
\hline Dialium guianense & $324,6 \mathrm{DE}$ & $48,0 \mathrm{~B}$ & $88,2 \mathrm{BC}$ & 629,9A & $1.062,2 \mathrm{~A}$ & 91,3АBC \\
\hline Thyrsodium schomburgkianum & 365,5CD & $70,2 \mathrm{~A}$ & $87,8 \mathrm{BC}$ & 358,2ABC & $1.398,1 \mathrm{~A}$ & 161,8A \\
\hline Tapirira guianensis & $254,8 \mathrm{~F}$ & $64,3 \mathrm{~A}$ & $60,0 \mathrm{DE}$ & 302,2ABC & $1.209,2 \mathrm{~A}$ & 72,9BC \\
\hline Lecythis pisonis & $171,0 \mathrm{G}$ & $42,2 B$ & $100,8 \mathrm{AB}$ & 360,0ABC & $944,5 \mathrm{~A}$ & $83,6 \mathrm{ABC}$ \\
\hline Schefflera morototoni & $277,6 \mathrm{EF}$ & $42,4 \mathrm{~B}$ & $26,8 \mathrm{~F}$ & $89,8 \mathrm{C}$ & $732,5 \mathrm{~A}$ & $61,6 \mathrm{BC}$ \\
\hline Média & 328,1 & 48,7 & 71,6 & 353,7 & $1.108,4$ & 91,4 \\
\hline DMS & 65,2 & 10,6 & 24,7 & 354,5 & 989,4 & 83,7 \\
\hline $\mathrm{CV}$ & 6,9 & 7,4 & 11,8 & 34,2 & 59,0 & 31,3 \\
\hline
\end{tabular}

Em que: Médias seguidas de mesma letra na coluna não diferem estatisticamente pelo Teste de Tukey a 5\%.

\section{CONCLUSÕES}

Os teores dos nutrientes nas folhas apresentaram a seguinte ordem decrescente $\mathrm{N}>\mathrm{Ca}>\mathrm{K}>\mathrm{Mg}>\mathrm{P}$, porém em algumas espécies o teor de $\mathrm{K}$ foi maior do que o de Ca.

A biomassa foliar das dez espécies de maior valor de importância foi de 3,3 tha ${ }^{-1}$ e representou 24,6\% da biomassa foliar total $\left(13,4 \mathrm{tha}^{-1}\right)$ do fragmento.

A espécie Schefflera morototoni foi a responsável pelo maior aporte via fração foliar de Ca e Mg e Helicostylis tomentosa pelo de N, P e K no fragmento.

A eficiência de utilização de nutrientes apresentou a seguinte ordem decrescente $\mathrm{P}>\mathrm{Mg}>\mathrm{K}>\mathrm{Ca}>\mathrm{N}$.

\section{AGRADECIMENTOS}

Ao Comando Militar do Nordeste, pela cessão da área onde foram instalados os equipamentos para coleta dos dados desta pesquisa.

\section{REFERÊNCIAS BIBLIOGRÁFICAS}

BALIEIRO, F. C. et al. Acúmulo de nutrientes na parte aérea, na serapilheira acumulada sobre o solo e decomposição de filódios de Acacia mangium Willd. Ciência Florestal, Santa Maria, v. 14, n. 1, p. 59-65, 2004.

BARROS, N. F. et al. Biomassa, absorção e eficiência de utilização de nutrientes por clones de eucalipto na região norte do Espírito Santo. In: CONGRESSO BRASILEIRO DE CIÊNCIA DO SOLO, 20., 1985, Belém. Anais... Belém, SBCS, 1985, p.109.

BATAGLIA, O. C. et al. Métodos de análise química de plantas. Campinas: Instituto Agronômico, 1983. 48 p.

BRAGA , J. M.; DEFELIPO, B. V. Determinação espectrofotométrica de fósforo em extrato de solo e material vegetal.

Revista Ceres, Viçosa, v.21, p.73-85, 1974.

CLARKSON, D. T. Factors affecting mineral nutrient aquisition by plants. Ann Rev. Plant Physiol., v. 36, p.77-115, 1985.

CLEVELÁRIO JUNIOR, J. Distribuição de carbono e de elementos minerais em um ecossistema florestal tropical úmido baixo-montano. 1996. 135 f. Tese (Doutorado em Ciência Florestal) - Universidade Federal de Viçosa, Viçosa. CRONQUIST, A. The evolution and classification of flowering plants. New York: The New York Botanical Garden, 1988. $555 \mathrm{p}$.

DRUMOND, M. A. Alterações fitossociológicas e edáficas decorrentes de modificações da cobertura vegetal na mata atlântica, Região do Médio Rio Doce, MG. 1996. 117 f. Tese (Doutorado em Ciência Florestal) - Universidade 
Federal de Viçosa, Viçosa.

DRUMOND, M. A.et al. Composição mineral e demanda nutricional de espécies florestais da mata atlântica, Viçosa, Revista Árvore, Viçosa, v. 21, n. 1, p. 1-10, 1997.

EMBRAPA. Manual de análises químicas de solos, plantas e fertilizantes. Brasília: EMBRAPA, 1999a. 370 p.

EMBRAPA. Sistema Brasileiro de Classificação de Solos. Brasília: EMBRAPA, 1999b. 412 p.

GOLLEY, F. B. et al. Ciclagem de minerais em um ecossistema de floresta tropical úmida. São Paulo: EPUEDUSP, 1978. 256 p.

GRAHAM, R. D. Breeding for nutritional characterístics in cereal. In: TINKER, P. B; LANCHLI, A. (Ed). Advances in plant nutrition. New York: Praeger Publishers, 1984. p.57-102.

GRUBB, P. J. Control of Forest growth and distribuition on wet tropical mountains: with special reference to mineral nutrition. Ann. Rev. Ecol. Syst., v. 8, p. 83-107, 1977.

IBGE. Instituto Brasileiro De Geografia E Estatística. Manual Técnico da Vegetação Brasileira, Rio de Janeiro, 1992. $123 \mathrm{p}$.

LINS e SILVA, A. C. B. Florística e fitossociologia do componente arbóreo em um fragmento de Mata Atlântica na Região Metropolitana do Recife/PE. 1996. 109 f. Monografia (Graduação em Biologia) - Universidade Federal Rural de Pernambuco, Recife.

MALAVOLTA, E.; VITTI, G. C.; OLIVEIRA, S. A. de. Avaliação do estado nutricional das plantas: princípios e aplicações. Piracicaba: Associação Brasileira para Pesquisa de Potassa e do Fosfato, 1989. 210 p.

MARANGON, L. C. Florística e fitossociologia de área de floresta estacional semidecidual visando dinâmica de espécies florestais arbóreas no município de Viçosa - MG. 1999. 139 f. Tese (Doutorado em Ecologia e Recursos Naturais) - Universidade Federal de São Carlos, São Carlos.

MOLICA, S. G. Produção de biomassa e eficiência nutricional de híbridos interespecíficos de eucalipto, em duas Regiões Bioclimáticas de Minas Gerais. 1992. 84 f. Tese (Doutorado em Fitotecnia) - Universidade Federal de Viçosa, Viçosa.

MOURA, O. N. Distribuição de biomassa, nutrientes e eficiência nutricional em povoamentos de sabiá (Mimosa caesalpiniifolia Benth.) cultivados em Podzólico Vermelho - Amarelo. 1999. 52 f. Dissertação (Mestrado em Ciência do Solo) - Universidade Federal Rural de Pernambuco, Recife.

OLIVEIRA, R. E. Aspectos da dinâmica de um fragmento florestal em Piracicaba-SP: silvigênese e ciclagem de nutrientes. 1997. 79 f. Dissertação (Metrado em Ciências/Ciências Florestais) - Escola Superior de agricultura "Luiz de Queiroz", Piracicaba.

OLIVEIRA, R. R. Produção e deposição de serapilheira no Parque Nacional da Tijuca, RJ. 1987. 107 f. Dissertação (Mestrado em Geografia) - Universidade Federal do Rio de Janeiro, Rio de Janeiro.

REIS, M. G. F; BARROS, N. F. Ciclagem de nutrientes em plantios de eucalipto. In: BARROS, N. F; NOVAIS, R. F. (Ed). Relação solo-eucalipto. Viçosa, Ed. Folha de Viçosa, 1990. p.265-301.

SAMPAIO, E. V. S. B.; NUNES, K. S.; LEMOS, E. E. P. Ciclagem de nutrientes na mata de Dois Irmãos (Recife-PE) através da queda de material vegetal, Brasília, Pesquisa Agropecuária Brasileira, Brasília, v. 23, n. 10, p. 1055-1061, 1988.

SCHUMACHER, M. V. et al. Biomassa e nutrientes em um povoamento de Hovenia dulcis Thun., plantado na Fepagro florestas, Santa Maria, RS. Ciência Florestal, Santa Maria, v. 18, n. 1e 2, p. 27-37, jan./jun. 2008.

SCHUMACHER, M. V.; CALDEIRA, M. V. W. Estimativa da biomassa e do conteúdo de nutrientes de um povoamento de Eucalyptus globulus (Labillardière) sub-espécie maidenii. Ciência Florestal, Santa Maria, v. 11, n. 1, p. 45-53, 2001.

SILVEIRA, N. D. et al. Aporte de nutrientes e biomassa via serrapilheira em sistemas agroflorestais em Paraty (RJ). Ciência Florestal, Santa Maria, v. 17, n. 2, p. 129-136, 2007.

VELOSO, H. P; GOES FILHO, L. Fitogeografia brasileira, classificação fisionômica ecológica da vegetação neotropical. Salvador: Projeto RADAMBRASIL, 1982. 80 p.

VITAL, A. R. T. et al. Produção de serapilheira e ciclagem de nutrientes de uma floresta estacional semidecidual em zona ripária. R. Árvore, Viçosa, v. 28, n. 6, p. 793-800, 2004.

VITOUSEK, P. M.; SANFORD JR., R. L. Nutrient cycling in most tropical forest. Ann. Rev. Ecol. Syst., v. 17, p.137167, 1986.

YOUNG, H. E.; CARPENTER, P. N. Sampling variation of nutrient element content within and between tree of same species. In: OSLO BIOMASS STUDIES, 1976, Oslo. Proceedings... Oslo: IUFRO, 1976. p.75-99. 